\title{
Anisotropic self-assembly from isotropic colloidal building blocks
}

\author{
Marcel Rey ${ }^{\mathrm{a}, \mathrm{b}}$, Adam D. Law ${ }^{\mathrm{c}, \mathrm{d}}$, D. Martin A. Buzza ${ }^{*}$, Nicolas Vogel ${ }^{\star \mathrm{a}, \mathrm{b}}$ \\ a Institute of Particle Technology, Friedrich-Alexander University Erlangen-Nürnberg, Cauerstrasse 4, 91058 Erlan- \\ gen, Germany \\ b Interdisciplinary Center for Functional Particle Systems, Friedrich-Alexander University Erlangen-Nürnberg, Ha- \\ berstrasse 9a, 91058 Erlangen, Germany \\ ${ }^{c}$ Max Planck Institute for Intelligent Systems, Heisenbergstrasse 3, 70569 Stuttgart, Germany \\ d IV. Institute of Theoretical Physics, University of Stuttgart, Pfaffenwaldring 57, 70569 Stuttgart, Germany \\ ${ }^{\mathrm{e}} \mathrm{G}$ W Gray Centre for Advanced Materials, School of Mathematics \& Physical Sciences, University of Hull, Hull \\ HU6 ${ }_{7}$ RX, United Kingdom
}

\begin{abstract}
Spherical colloidal particles generally self-assemble into hexagonal lattices in two dimensions. However, more complex, non-hexagonal phases have been predicted theoretically for isotropic particles with a soft repulsive shoulder but have not been experimentally realized. We study the phase behaviour of microspheres in the presence of poly $(\mathrm{N}$ isopropylacrylamide) (PNiPAm) microgels at the air/water interface. We observe a complex phase diagram, including phases with chain and square arrangements, which exclusively form in the presence of the microgels. Our experimental data suggests that the microgels form a corona around the microspheres and induce a soft repulsive shoulder that governs the self-assembly in this system. The observed structures are fully reproduced by both minimum energy calculations and finite temperature Monte Carlo simulations of hard core-soft shoulder particles with experimentally realistic interaction parameters. Our results demonstrate how complex, anisotropic assembly patterns can be realized from entirely isotropic building blocks by control of the interaction potential.
\end{abstract}

\section{Introduction}

The ability of colloidal particles to adsorb to liquid interfaces is of fundamental importance for a range of scientific disciplines and applications ${ }^{1,2}$. The presence of the particles modifies the properties of the interface ${ }^{1}$, imparting for example mechanical and kinetic stability to emulsions ${ }^{3,4}$ or foams 5,6 .

Similarly, liquid interfaces confine particles in two dimensions and serve as ideal templates to observe their interactions and dynamics ${ }^{1,7,8}$. In contrast to atomic crystals where atoms interact via complex and predetermined potentials, colloidal interaction can often be described by simpler interaction potentials, which can be more readily controlled at the particle level or via external forces ${ }^{9-11}$. These increased degrees of freedom, and the ability to directly observe the phase behaviour with an optical microscope provide an ideal model system to fundamentally study self-organization phenomena.

If the size polydispersity is sufficiently low, colloidal particles are able to form two-dimensional crystals with longrange order ${ }^{10}$. This property, coupled with the ability to control the periodicity via the size of the particles, provides an experimentally simple, bottom-up strategy to create nanoscale surface patterns with high fidelity over macroscopic areas, exploited in photonic ${ }^{12,13}$, phononic ${ }^{14}$ or lithographic ${ }^{15,16}$ applications. However, control over the available symmetries of such two-dimensional colloidal crystals is currently limited.

The geometry of the colloidal building blocks generally determines the symmetry of the resulting assembly structure. The simplest and most widely used building blocks are charged-stabilized spherical polymer or silica particles. As expected intuitively from macroscopic objects, such spherical building blocks form crystals with hexagonal symmetry when placed at an air/water interface. Depending on the balance of attractive capillary and vander-Waals forces and electrostatic repulsion, the particles can either form close-packed, dense structures or nonclose-packed lattices where the interparticle distance is maximized ${ }^{17}$. However, the symmetry of the assembly remains hexagonal as the particles maintain an equal distance to all their neighbours ${ }^{18}$.

As a consequence of the isotropic nature of particle and interaction potential, the formation of two-dimensional crystals with different symmetry or anisotropic structures appears to be prohibited with spherical particles. Experimental efforts have therefore focused on more complex approaches, employing different building blocks, external stimuli or manipulations of the confinement. Cubic or octahedral particles tend assemble into square packing, which is their densest packing ${ }^{19,20}$. Alternatively, external electric ${ }^{21-23}$ or magnetic fields ${ }^{24-26}$, inducing dipole moments in the particles, can direct spherical particles into anisotropic chain structures. Manipulating the direction- 
ality of capillary forces, using anisotropic particles ${ }^{27,28}$, a defined curvature of the liquid interface ${ }^{29,30}$ or the introduction of defined patches on isotropic particles ${ }^{31}$ provides another tool to guide the assembly process towards chain or square structures. Common to all these approaches is the introduction of an anisotropic component into the interaction potential, which provides energetically favourable sites at defined positions at a particle's vicinity, thus directing the assembly process.

An elegant, albeit less intuitive route towards anisotropic assemblies was theoretically predicted by Jagla nearly two decades ago ${ }^{32,33}$. Jagla showed that spherical particles confined to two dimensions and possessing an isotropic and repulsive interaction potential can form anisotropic assemblies as their minimum energy structure if the interaction potential features two distinct length scales. The combination of a hard-sphere potential acting at the particle core with a longer range soft repulsion shoulder gives rise to a variety of different equilibrium structures at different surface densities, including - counterintuitively for purely spherical building blocks - chains and square symmetries as the system minimizes its energy by fully overlapping the shells of neighbouring particles in some directions in order to prevent the overlap of the shells in others 29,32,34. Recently, even more complex structural motifs such as quasicrystalline order has been predicted via similar potentials ${ }^{35-37}$. Importantly, theoretical investigations suggest the possibility to induce complex, anisotropic assembly patterns from entirely isotropic colloidal particles $^{38,39}$. However, even though the minimum energy structures above are recovered by Monte Carlo simulations ${ }^{36,40}$, indicating a robust phase that can be potentially experimentally accessible, no experimental evidence of a phase diagram reproducing the essential features predicted by the soft-repulsion models has been demonstrated for core-shell particles to date.

Here, we explore the phase diagram of polymer polystyrene (PS) microspheres at the air/water interface in the presence of soft poly(N-isopropylacrylamide) (PNiPAm) microgels with nanoscale dimensions. PNiPAm microgels are ideal model systems to introduce a soft character since they are able to swell by taking up large amounts of water and to significantly deform at the air/water interface ${ }^{41,42}$. We show that the attractive interactions between the two particle populations lead to the in-situ formation of a soft, compressible microgel corona around the polystyrene microspheres so that our binary system effectively acts as a one component core-shell system. Upon increasing the density of the particles, we observe a complex phase diagram of assembly structures of the microspheres, including chain and square phases. The experimental data is in excellent agreement with minimum energy calculations and finite temperature Monte Carlo simulations of hard core-soft shoulder particles with experimentally realistic interaction parameters. Our results provide the first experimental realization of complex, anisotropic assembly patterns produced from entirely isotropic particles and interactions with a soft-repulsive component in the interaction potential.
Results

\section{Particle interactions}

To investigate the phase behaviour of the binary mixture of polystyrene (PS) microspheres (diameter $\mathrm{d}=1.5 \mu \mathrm{m}$, Supplementary Figure 1) with PNiPAm microgels ( $d=145$ nm, Supplementary Figure 2), we premixed the two particle populations prior to spreading at the air/water interface of a Langmuir trough (Figure 1a). Even though both the PS microspheres as well as the PNiPAm microgels are negatively charged, we observe attractive interactions between the two particle populations, both using fluorescently labelled microgels in a confocal microscope (Figure 1b,c) and by scanning electron microscopy (Figure 1d,e), in agreement with previous reports 43,44 .

To ensure a complete microgel shell and to avoid aggregation caused by bridging of microspheres via the microgels at low concentrations 43 all experiments were performed with an excess of microgels. Importantly, this implies free microgels in the dispersion and at the interface.

The presence of microgels seemingly affects the interaction potential between the large microspheres. For pure microspheres, we observed close-packed clusters even at very low surface pressures $(\pi<1 \mathrm{mN} / \mathrm{m})$ (Figure $2 \mathrm{~b})$, which typically form at air/water interfaces because of capillary attraction ${ }^{17}$ (Van der Waals attractive forces are too short ranged to be relevant on the length scales we are considering here). In the presence of microgels, a non-close packedarrangement resulted instead (Figure 2c). We did not observe any indications of phase separation between microgels and microspheres, which suggests a preferential, attractive interaction between microgels and microspheres, consistent with their interactions in bulk (Figure 1). Furthermore, the PS microspheres in the presence

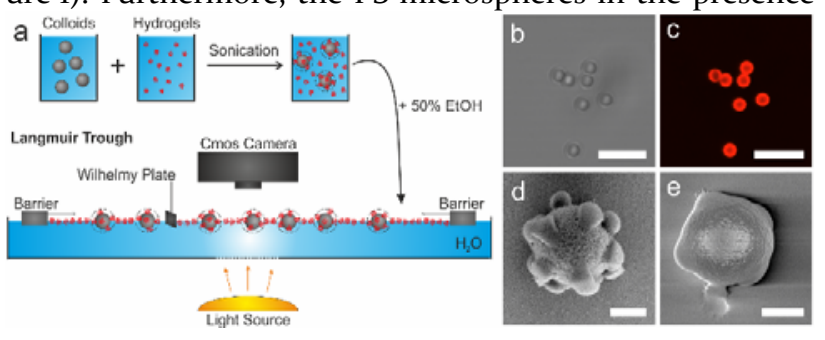

Figure 1. Behaviour of mixtures of polystyrene (PS) microspheres and poly(N-isopropylacrylamide; PNIPAm) microgels and experimental setup. a) Schematic illustration of the experiment: PS microspheres $(\mathrm{d}=1.5 \mu \mathrm{m})$ are mixed with PNIPAm microgels $(\mathrm{d}=150 \mathrm{~nm})$, sonicated and then spread at the air/water interface on a Langmuir trough to observe the phase behaviour upon compression. b,c) Confocal microscopy images of PS microspheres mixed with fluorescentlylabelled PNIPAm microgels. The position of the microspheres, seen in bright field (b) coincides with the position of the labelled microgels, seen in the fluorescence image (c), indicating adsorption of the microgels onto the microspheres. Scale bars are $5 \mu \mathrm{m}$. d,e) SEM image of a mixture of PS microspheres and larger PNIPAm microgels $(d=550 \mathrm{~nm})$ deposited at $50^{\circ} \mathrm{C}(\mathrm{d})$ and $23^{\circ} \mathrm{C}(\mathrm{e})$, providing direct evidence of the microgel adsorption onto the PS particles. The difference in morphology between collapsed state (d) and swollen state (e) is clearly visible. Scale bars are $500 \mathrm{~nm}$. 
of microgels did not show observable Brownian motion as soon as the surface pressure started rising $(\pi>3 \mathrm{mN} / \mathrm{m})$, even though the microspheres were not in close contact, demonstrating that the microgels introduce an additional long-range repulsive component to the interaction potential between the microspheres (greater than $k_{B} T$ ). The fact that we do not observe any close-packed clusters in Figure $2 \mathrm{c}$, $\mathrm{d}$ also suggests that this microgel-induced repulsion is much stronger than the capillary attraction between latex spheres. Note that the addition of microgels does not lead to 'depletion attraction' between the latex particles. Instead, the attractive microgel-colloid interactions in our system leads to the opposite case of 'accumulation repulsion' between the latex particles, as discussed in detail in the discussion below 45 .

To probe the nature of this repulsion in greater detail, we consider the surface pressure-area isotherms in Figure 2 a. In the absence of microgels, no increase in surface pressure is recorded until the microspheres form a dense monolayer, upon which the isotherm rises steeply as a result of the increased stiffness ${ }^{46}$. In the presence of microgels, the surface pressure rises at much lower compressions and more gradually as a result of the softness and compressibility of the microgels that interact over longer distances ${ }^{47}$. The composite microspheres/microgel system showed a similar behaviour as pure microgels (Figure 2a; Supplementary Figure 3), indicating that the mixed system is dominated by the elasticity of the microgels. At high compressions $(\pi>28.5 \mathrm{mN} / \mathrm{m})$, the steep rise in surface pressure indicates that the microspheres in close contact dominate the interfacial behaviour. We further note that upon spreading of pure microspheres, a fraction did not adsorb to the air/water interface and submerged to the subphase, while in the mixed sample, no microspheres in the subphase were observed. This behaviour is further evidence of the adsorption of microgels onto the PS microspheres' surface, which seemingly increases their surface activity.

To investigate the phase behaviour of the PS microspheres in the presence of the microgels, we compressed the interface until the microspheres were in contact $(\pi=34 \mathrm{mN} / \mathrm{m})$. We note that the isotherm of pure microgels did not increase above $\pi=28.5 \mathrm{mN} / \mathrm{m}$ upon further compression, regardless of the initial concentration of microgels (Supplementary Figure 3), indicating that microgels detached from the interface at higher compressions. We equilibrated the system via relaxation/compression cycles (stopping at $\pi=34 \mathrm{mN} / \mathrm{m}$ to avoid collapse of the microsphere layer) to remove excess microgels from the interface. After 3 cycles, the shape of the surface pressure-area isotherm did not change any further (Supplementary Figure 4), indicating that the particle concentration and composition at the interface remained constant. We used this equilibrated system to investigate the phase behaviour as a function of particle density. Importantly, as the equilibration step removes excess microgels from the interface, the system is insensitive to the initial concentration of microgels. Indeed, we
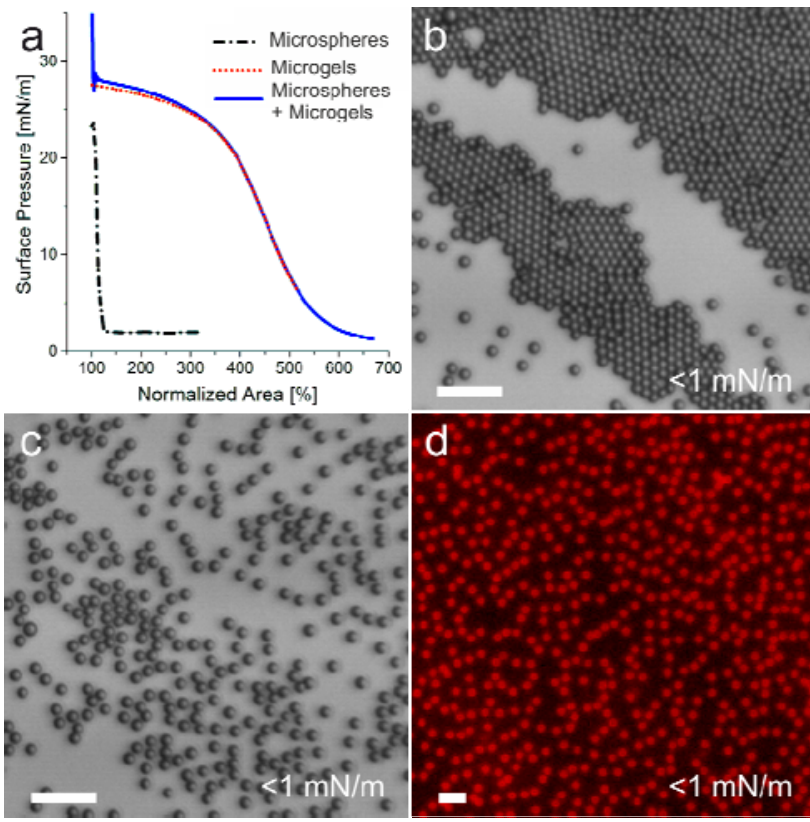

Figure 2. Effect of microgel addition on interfacial properties of PS microspheres. a) Compression isotherm of pure PS microspheres (black), the mixed suspension (blue) and of pure microgels (red). The interfacial behaviour of the mixed system is dominated by the microgels. b) Pure PS microspheres form close-packed clusters at the air/water interface. c) In the presence of microgels (fluorescently labelled, mass ratio of microgels/microspheres $=4 \mathrm{wt}-\%$ ), a non-close packed arrangement of PS microspheres is observed. d) Fluorescence microscopy image showing the selective adsorption of microgels in a corona around the microspheres. Scale bar: $10 \mu \mathrm{m}$.

observed identical isotherms and phase diagrams for higher initial microgel concentrations (Supplementary Figure 5). However, at lower initial microgel to microsphere ratios ( $1 \mathrm{wt}-\%)$, we observed the formation of aggregated microsphere structures at the interface (Supplementary Figure 5) similar to pure microspheres, indicating insufficient microgels at the interface to induce changes in the interaction potential. We conclude that the attraction of the microgels to the microspheres introduces an effective repulsion between the microspheres and that a minimum concentration of microgels is necessary to prevent the formation of close-packed structures and to observe the complex phase diagram of the composite system. We note that the characteristic distances between microspheres in Figure 2 and reported later in Figure 3 exceed the diameter of a single microgel particle. This large length scale cannot be accounted for by the single layer of microgels that are adsorbed around the microsphere in the bulk dispersion alone (see Figure 1), and suggests the presence of additional microgels in the vicinity of the microspheres, even after equilibration.

As we discuss below, we interpret this behaviour in the context of the formation of a soft, two-dimensional shell around the microspheres that forms in situ at the interface and induces a soft, repulsive component. Direct evidence of the formation of this microgel corona can be 
seen in Figure $2 \mathrm{~d}$ that shows a microscopy image taken at the air/water interface with fluorescently-labelled microgels, mixed together with polystyrene microspheres (same experimental setup as in c). Clearly, the microgels arrange around the polystyrene microspheres. Since all particles are confined to the air/water interface, we deduce that the corona of microgels at the interface must be effectively two-dimensional.

Phase diagram as a function of the available area.

We compressed the system until we observed a hexagonally close-packed phase at $\pi=34 \mathrm{mN} / \mathrm{m}$ and slowly reduced the density of the particles at the interface by barrier expansion while recording the interfacial arrangement of the microspheres (Supplementary Movie 1) and use image analysis to extract position and number of particles. We can thus present the interfacial behaviour as the area per particle as a function of the surface pressure $\pi$ (Figure $3 \mathrm{a}$ ) or as the average distance of neighbouring particles as a function of the area per particle (Figure $3 \mathrm{~b}$ ). Our experiments revealed the presence of four distinct phases occurring at different surface pressures, as indicated in the insets of Figure 3a,b. Figure $3 c$-h shows representative microscopy images of the observed phases. At highest surface pressure - or highest surface density for the particles - we observed a hexagonal close-packed phase as expected from spherical, isotropic particles at the air/water interface ${ }^{17}$ (Figure $3 c$ ). Upon relaxation and thus increase of the area available per particle, the particle monolayer first relaxed into a phase with square symmetry (Figure 3d), followed by distinct particle chains, which became dominant once the area per particle exceeded $5 \mu^{2}$ (Figure $3 \mathrm{f}$ ). Subsequently, the chains gradually dissolved (Figure 3g) and gave rise to a non-close packed arrangement with increasing particle distances (Figure $3 \mathrm{~h}$ ). The different phases can be clearly distinguished from the interparticle distances in dependence of the available area distance) while the distance to other particles (e.g. in the neighbouring chain) is gradually,
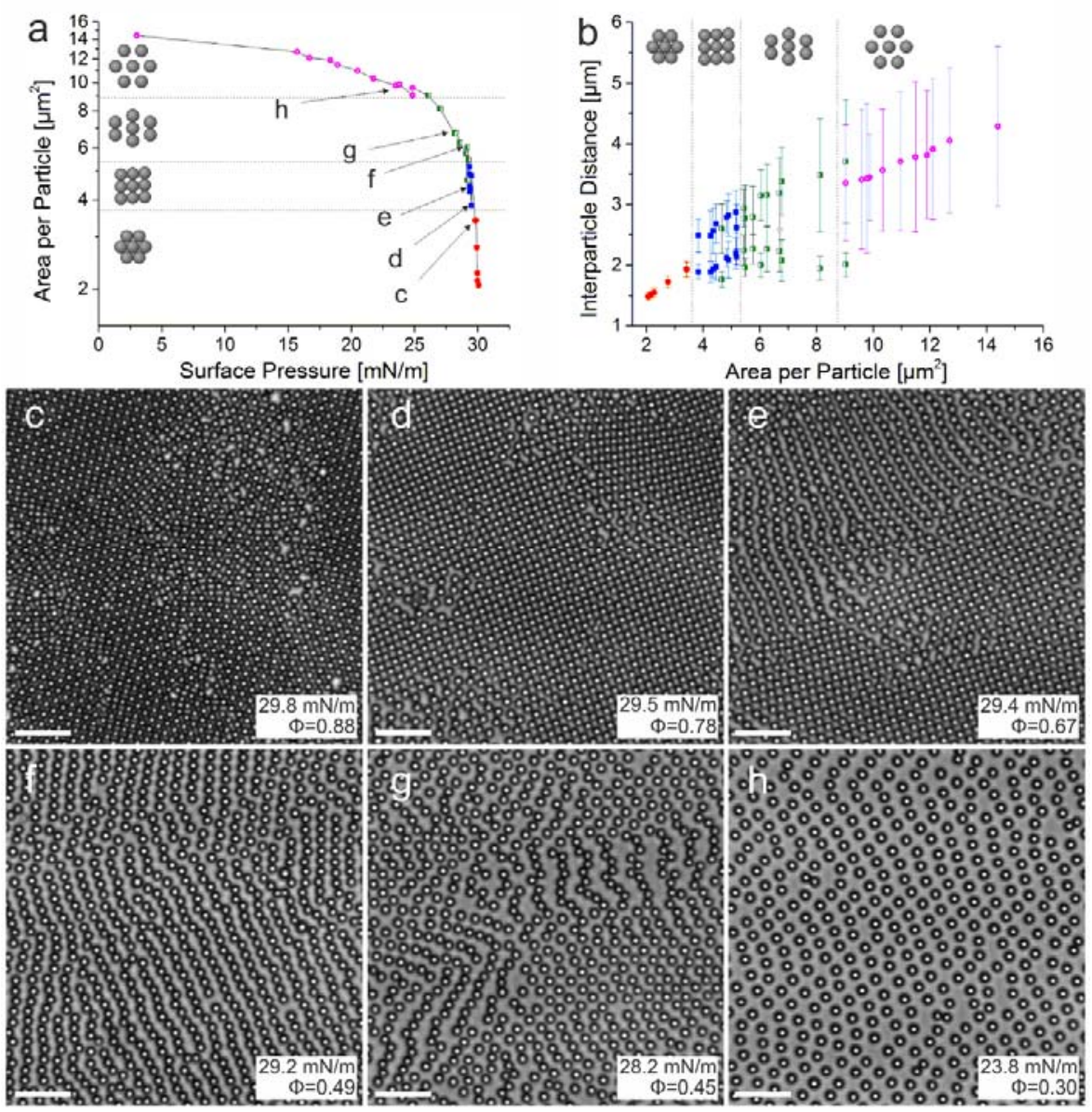

Figure 3. Phase diagram of a mixture of polystyrene microspheres $(\mathrm{d}=1.5 \mu \mathrm{m})$ and PNiPAm microgels $\left(\mathrm{d}_{\mathrm{H}}=150 \mathrm{~nm}\right)$ observed in situ at the air/water interface. a) Compression isotherm, represented as the surface pressure vs. area per particle extracted from image analysis. The insets indicate the different observed phases. b) Phase diagram represented as the nearest neighbour interparticle distance vs area per particle. The different phases are clearly distinguished by the bimodal character of the rhombic and chain phase. c-h) Representative microscopy images of the observed phases: c) Hexagonal close packed phase; d) Square phase; e) Transition between square and chain phase; f) Chain phase; g) Transition between chain phase and hexagonal nonclose packed phase; h) Hexagonal non-close packed phase. All scale bars are $10 \mu \mathrm{m}$. 
increased with increasing area per particle. In contrast both hexagonal phases are characterized by a monomodal nearest neighbour distribution, enabling to clearly map out the phase diagram. To facilitate comparison with theoretical results, we also specify the area fraction $\phi=$ $\rho \pi r_{o}^{2} / 4$, where $\rho$ is the number of particles per unit area and $r_{0}=1.95 \pm 0.2 \mu \mathrm{m}$ is the minimum distance between neighbouring particles in the densest phase, which we interpret as the closest possible packing, or core diameter, of the system (Figure $3 \mathrm{~b}$ ). This minimum distance $r_{0}$, extracted from statistical image analysis, remained constant throughout the phase transitions from hexagonal close packed to square and chains. By comparison with our theoretical model (see below), we therefore attribute this to the core diameter of the system. Importantly, we realize that this "core" consists of the microsphere with a highly compressed microgel corona. We estimate the extend of this corona by measuring the space required for individual microgels at a similar surface pressure and find a value of approximately $160 \mathrm{~nm}$ (Supplementary Figure 6). By substracting the actual microsphere diameter from the area covered by a particle with the experimental core diameter, we can estimate the number of microgels within the shell, and determine value of approximately 58 microgels around the microspheres (Supplementary Figure 6). In agreement with the accumulation repulsion model (details below), this indicates multiple microgel layers surrounding the central microsphere.

For all the phases, we observed a transition with coexistence of two different phases (Figure 3e,g). Additionally, the crystal domain structure initially present in the hexagonal close packed phase persisted throughout the phase transitions. The orientation of the crystal domains with respect to the barrier position seemed to influence the phase transition behaviour. In Figure $3 \mathrm{e}$, the crystal domain at the top left, oriented nearly parallel to the barriers and thus the direction of relaxation (the barriers opened from left and right of the image) already formed the chain phase, while the domain at the bottom, oriented diagonal to the barriers still remained in the square phase. We attribute this behaviour to the anisotropic nature of the relaxation. The transition from squares into the chain phase occurs by anisotropic relaxation of the crystal domains perpendicular to the chain orientation, therefore requiring more space along the direction of the expansion. As the barrier opening occurs along one axis (left to right in the images), the phase transition occurs preferentially along this direction as well, leading to a preferential alignment of the chains parallel to the barriers, which is clearly observed in Figure 3 f. The transition from chains into hexagonal non-close packed phase though requires melting of the particle chains and nucleates at the grain boundaries (Figure 3g), similar to what has been observed for bulk colloidal crystals ${ }^{48}$.

Importantly, all experiments were started at maximum particle density. As the particles gain more area at the interface, they relax and the system evolves into different, distinct phases before finally transitioning in a non-close packed arrangement. This behaviour indicates a fully reversible process (indeed, repeated cycles gave identical phase behaviour, Supplementary Figure 7) resulting in phases with identical length scale. The reversibility and constant length scale in the assembly structures, in corroboration with identical surface pressure-area isotherms indicates a constant ratio of microspheres and microgels, which suggests that the microgels form a stable, twodimensional shell around each microsphere.

When recording the phase diagram from low to high compression, the system also underwent phase transitions (Supplementary Figure 7). The individual phases were, however, much less defined and regular, indicating that kinetic trapping and jamming can obscure the observation of the phases.

\section{Characterization of the different phases}

In Figure 4, we present a detailed characterization of the observed phases. From the microscopy images taken of the microspheres at the air/water interface (Figure 4a-d), we extracted the position of each particle and used a Voronoi tessellation to determine their nearest neighbours. We characterized the different phases by Fourier transform analysis (Figure 4a-d, insets), by the nearest neighbour distance (Figure 4e-h), by the angular distribution of the all neighbours (Figure $4 \mathrm{i}-\mathrm{l}$ ) and of neighbouring particles in direct contact (Figure $4 \mathrm{~m}-\mathrm{p}$ ).

For the hexagonal close-packed phase, we see a sharp peak at $1.95 \pm 0.2 \mu \mathrm{m}$ in the nearest neighbour distribution, which is the minimum distance between the microspheres in our system and we accordingly correlate with the hard sphere (or core) diameter $r_{\mathrm{o}}$ (Figure 4e). The difference between this measured diameter and the nominal diameter of the microspheres $(\mathrm{d}=1.5 \mu \mathrm{m})$ indicates the presence of compressed microgels around the microspheres, even at maximum compression. The hexagonal symmetry of the phase is reflected by the Fourier transformation pattern (Figure 4a) and the $60^{\circ}$ angles between nearest neighbours (Figure $4 \mathrm{i}$ ). The square phase shows a clear four-fold symmetry in the Fourier transformation (Figure $4 \mathrm{~b}$ ) and is characterized by a bimodal distribution of nearest neighbours, related to neighbouring particles in direct contact and neighbours sitting at the diagonal position (Figure $4 \mathrm{f}$ ). The position of the first peak remains unchanged at $1.95 \mu \mathrm{m}$ (red), indicating that microspheres remain in close contact. The second peak appears at 2.6 $\pm 0.2 \mu \mathrm{m}$ (blue), which is approximately $\sqrt{2}$ times the first distance (Figure $4 \mathrm{f}$, inset). The two characteristic length scales are further reflected in the angular distribution of all nearest neighbours that shows a similar bimodality with peaks at $86^{\circ}$ and $47^{\circ}$ in a ratio of 1:2 (Figure $4 \mathrm{~g}$ ), corresponding to a rhombus with internal angles of $86^{\circ}$ and $94^{\circ}$, indicating that the squares are slightly distorted into a rhombic structure. Particles in core contact show a clear peak around $90^{\circ}$ (Figure $4 \mathrm{n}$ ), with a minor peak at $60^{\circ}$ corresponding to particles remaining in the 

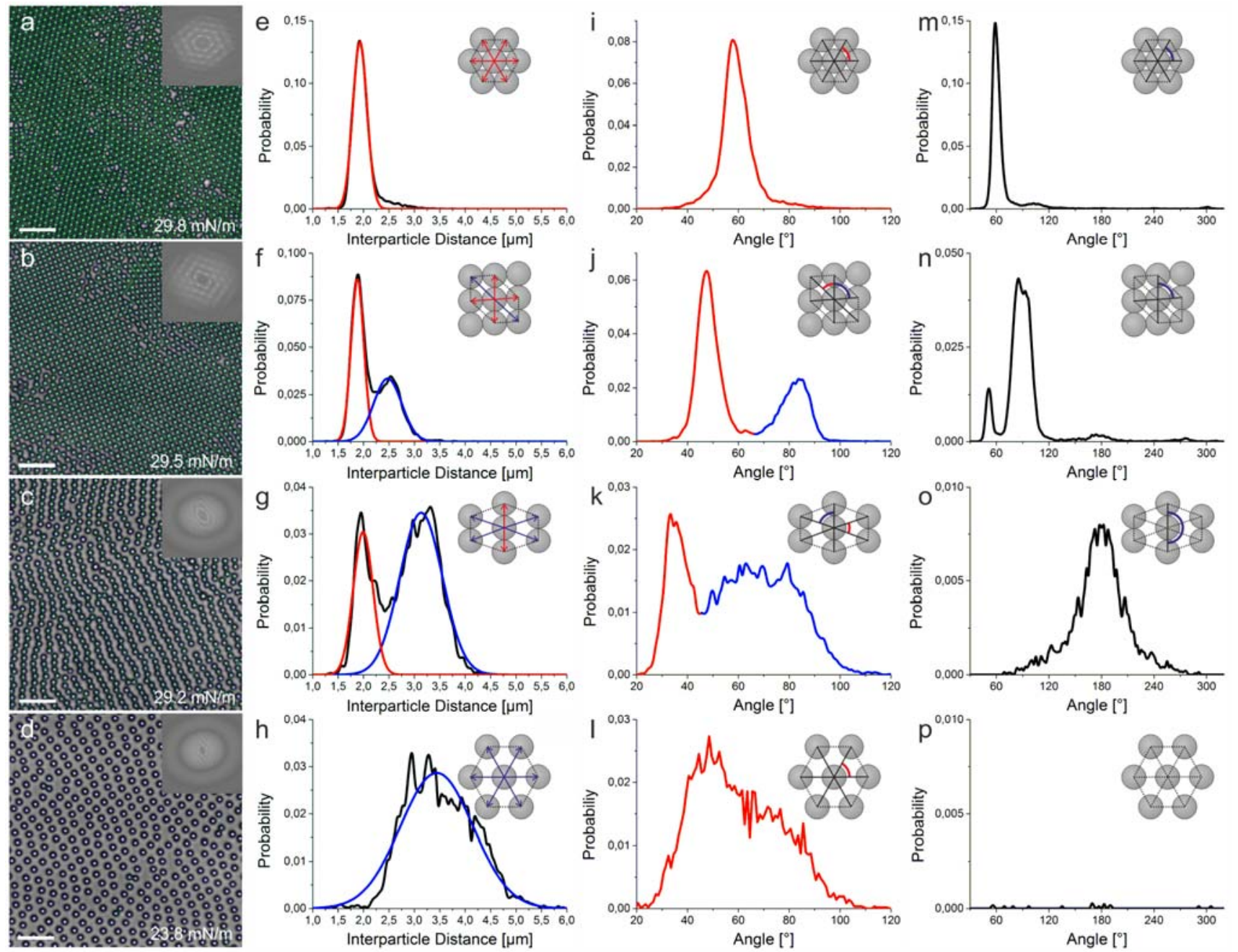

Figure 4: Statistical image analysis of the observed phases. a-d) Microscopy images of each phase analysed by customized particle tracking code. Particles in close contact are marked as green lines. Fourier transformation is shown as inset. All scale bars are $10 \mu \mathrm{m}$. e-h) Interparticle distance distribution, revealing the prevalence of a peak at $1.95 \mu \mathrm{m}$ throughout the hexagonal closepacked, square and chain phase, attributed to particles in core contact. A bimodal distribution is found for the rhombic and chain-like phase, reflecting the anisotropy of the crystal structure. i-l) Angular distribution between the nearest neighbours with assignment of the angles shown as insets. m-p) Angular distribution between particles in core contact (defined as the contacts of the peak at $1.95 \mu \mathrm{m}$ in the interparticle distance diagram). Scale bar: $10 \mu \mathrm{m}$.

hexagonal close-packed phase. The anisotropy of the chain phase, clearly observable in the Fourier transformation pattern (Figure $4 \mathrm{c}$ ), is reflected in the nearest neighbour distribution (Figure $4 \mathrm{~g}$ ). The first peak remains at $1.95 \mu \mathrm{m}$ and demonstrate core-core contact of particles within the chain. A second, broad peak centred around $2.95 \mu \mathrm{m}$ relates to particles in the neighbouring chains. The agngular distribution of all neighbours (Figure $4 \mathrm{k}$ ) shows a peak at $34^{\circ}$ and a broadened peak around $70^{\circ}$, indicating that there is a preferred, although not pronounced orientation of neighbouring chains in a hexagonal packing (i.e. particle position shifted by half the diameter in neighbouring chains, see inset). Particles in core contact form a clear angle of $180^{\circ}$, underlining the presence of parallel, aligned chains (Figure 40).
The hexagonal non-close packed shows a less defined packing (Figure 4d) and, consequently, much broader distributions of nearest neighbour distances and angle distributions (Figure $4 \mathrm{~h}, \mathrm{l}$ ). Importantly, and in contrast to the same microspheres in the absence of microgels (Figure $2 \mathrm{~b}$ ), no direct contact between the particles exists.

\section{Theoretical investigations}

To rationalize the influence of the microgels on the phase behaviour of microspheres, we assume a disk-like soft shell of microgels around each microsphere and modelled the interaction between microspheres using the generic hard core-soft shoulder potential proposed by Jagla ${ }^{32}$, 


$$
U_{g}(r)= \begin{cases}\infty & r<r_{0} \\ U_{0} \frac{g+\left[\left(\frac{r-r_{0}}{r_{1}-r_{0}}\right)\left(g-g^{-1}\right)-g\right]^{-1}}{g-g^{-1}} & \\ 0 & r_{0} \leq r \leq r_{1} \\ 0 & r>r_{1}\end{cases}
$$

where $r_{0}, r_{1}$ are the range of the core and shoulder repulsion respectively, $U_{o}$ is the shoulder height and the parameter $g$ controls the profile of the soft shoulder, going from no shoulder $(g=0)$, via a linear ramp potential $(g=1)$ to a square shoulder $(g=\infty)$ as we increase $g$ (Figure $5 \mathrm{a}$ ). The Jagla potential is fully described by three parameters, $r_{o}, r_{1}, U_{\mathrm{o}}$ and $g$, and we will determine their effective values by comparing the theory with experiment. We first study the zero temperature structures (i.e., the minimum energy configurations MECs) formed by particles interacting via the Jagla potential in two dimensions, resembling the particles confined to the air/water interface. The zero temperature regime is relevant to our system since we assume the interaction potential due to the microgel layer to be greater than the thermal energy, i.e., $U_{o}>>k_{b} T$, or equivalently the reduced temperature $T^{*}=T /\left(U_{o} / k_{b}\right)$. We perform a comprehensive exploration of all twodimensional structures containing one particle per unit cell (Figure $5 \mathrm{~b}$ ), compared to the original study by Jagla which only considered a limited

subset of MEC structures ${ }^{32}$. We use an NPT where the system can transform completely into a single phase and determine its MEC as a function of the parameters $r_{0}, r_{1}$, $g$, and $P$ (the parameter $U_{o}$ is irrelevant at zero temperature) by minimising the enthalpy per particle $H$ with respect to the lattice parameters (Methods).

We estimate the potential range from the experimental data (Figure 4 ) and use $r_{0}=1.95 \pm 0.2 \mu \mathrm{m}$ as the core repulsion length and the length scale of the soft repulsion shoulder $r_{1}=2.95 \pm 0.3 \mu \mathrm{m}$ from the interparticle distance in the chain phase, yielding a $r_{0} / r_{1}=1.5 \pm 0.15$.

Figure $5 \mathrm{c}, \mathrm{d}$ shows the phase diagram in the plane of $g$ and reduced pressure $P^{*}=r_{o}{ }^{2} P / U_{o}$ and the corresponding MECs structures, respectively. For $g>1$ the hexagonal phase at low pressures corresponds to the low density hexagonal phase (HEX-L) (no overlap of corona) and at high pressure to the high density hexagonal phase (HEX-H) (full overlap of corona). We note also that the phase diagram is relatively insensitive to $g$ when $g \geq 1$, with the same sequence of phases being observed as we increase $P^{*}$, going from HEX-L to chains $(\mathrm{CH})$, to the rectangular phase (REC) and finally to HEX-H. Importantly, since the chain phase only exists for $g \geq 1$, we conclude that $g \geq 1$ in our experimental system. For $g<1$, the repulsive shoulder is concave enough so that partial overlap of the corona becomes energetically favourable, allowing other phases such as rhomboidal (RH) and square (SQ) phases to exist. For even lower values of $g(g<0.6)$, the hexagonal phase

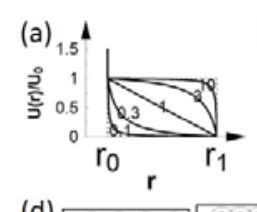

(b)

(d)

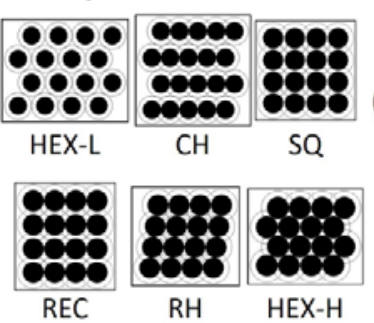

$2(c)$

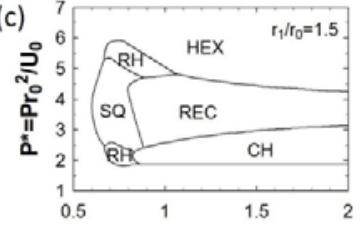

g
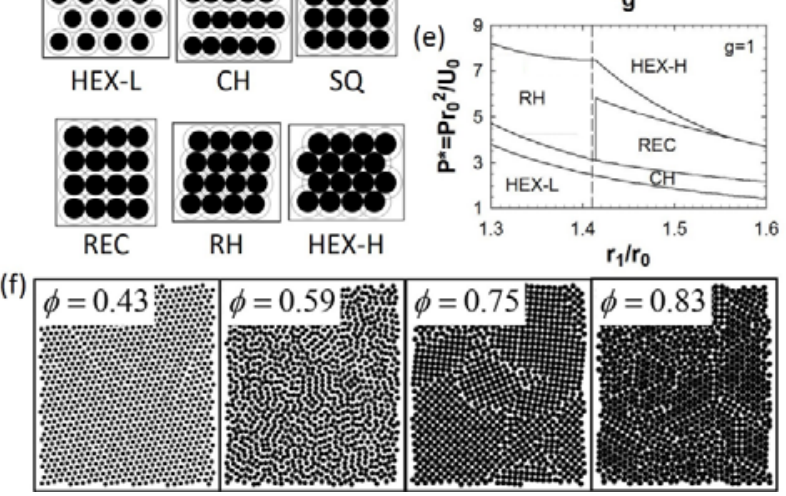

Figure 5. Theoretical modelling of particle interactions and resulting phase behaviour (a) Jagla potential for different values of $g$. The dotted lines on the left and upper right correspond to $g=0$ (no shoulder) and $g=\infty$ (square shoulder) respectively. (b) Unit cell used in our zero temperature calculations of two-dimensional structures, where $\boldsymbol{a}, \boldsymbol{b}$ are the lattice vectors, $\phi$ is the unit cell angle and the thick and thin circles represent the particle core and corona, respectively. (c) Zero temperature phase diagram for core-shell particles with $r_{1} / r_{0}=1.5$ in the $g$ and reduced pressure $P^{*}$ plane; (d) Representative minimum energy configurations (MECs) for $r_{1} / r_{0}=1.5$. (e) Zero temperature phase diagram for core-shell particles with $g=1$ in the $r_{1} / r_{o}-P^{*}$ plane. The vertical dashed line $\left(r_{1} / r_{0}=1.41\right)$ are the state points for which we performed finite temperature Monte Carlo simulations. The rhomboid MEC for $r_{1} / r_{o}=1.41$ is close to a square, with $\phi=90.3^{\circ}$; (f) Snapshots from NVT Monte Carlo simulations of core-shell particles with $r_{1} / r_{0}=1.41, g=1$ at a reduced temperature $T^{*}=0.01$ and different area fraction $\phi=\rho \pi r_{o}{ }^{2} / 4$. The solid circles represent the particle cores. Note the close correspondence of the area fraction in the MC simulations with the experimental data in Figure $3 \mathrm{c}-\mathrm{h}$.

goes continuously from HEX-L to HEX-H without any additional phases. The overall shape of the phase diagram qualitatively agrees with that calculated by Jagla 33 , with two noteworthy differences. Firstly, the region occupied by the rectangular phase (REC) in Figure $5 c$ broadly coincides with the region occupied by more complex MEC structures containing more particles per unit cell considered by Jagla, suggesting that the rectangular phase acts as a reasonable one-particle approximant for these more complex MECs. Secondly, while the square phase (SQ) extends considerably into the $g>1$ region in Jagla's phase diagram, in Figure $5 \mathrm{c}$ it is confined to the $g<1$ region. We believe that this discrepancy is due to the incomplete set of MECs used by Jagla which can lead to inaccuracies in the phase diagram 33. The implication is that for $r_{1} / r_{0}=1.5$, it is impossible to find any value of $g$ which reproduces 
the experimentally observed transition from chains to square-like rhombic structures with increasing pressure.

However, we note that the experimental uncertainty in $r_{1} / r_{o}$ is relatively large $\left(r_{1} / r_{0}=1.5 \pm 0.15\right)$ and that the phase diagram is very sensitive to the precise value of $r_{1} / r_{0}$ as illustrated in Figure 5e for the $r_{1} / r_{o}-P^{*}$ plane and $g=1$, where we focus on $r_{1} / r_{o}$ values within the experimental uncertainty (the motivation for choosing $g=1$ will be discussed below). The phase behaviour clearly changes considerably even within this small range of $r_{1} / r_{o}$ values. In particular, we note that when $r_{1} / r_{0} \rightarrow \sqrt{2}$, both the rectangular phase and the rhomboid phase approach the square structure for simple geometric reasons. Therefore, assuming $r_{1} / r_{o} \approx \sqrt{2}$, (dashed line in Figure $5 \mathrm{e}$ ), our MECs reproduce the experimentally observed phases and, with increasing pressure, evolves from low density hexagons, to chains, to rhombic structures with a quasi-square symmetry to high density hexagons.

In order to illustrate the close correspondence between theory and experiment more clearly, we performed finite temperature Monte Carlo simulations along the dashed line in Figure 5e (i.e., $r_{1} / r_{o}=1.41, g=1$ ). The simulations were performed at a reduced temperature of $T^{*}=0.01$ as this represents a good compromise between satisfying the experimental requirement that $U_{o}>>k_{B} T$ while still being computationally accessible through a slow quench from high temperature. We used the NVT ensemble in our simulations to mimic the experimental situation where we control monolayer area rather than surface pressure, allowing for two phase coexistence. Figure $5 \mathrm{f}$ shows representative structures formed at different area fraction : with increasing density, the system undergoes a transition from low density hexagons, to chains, squares and finally to a coexistence of squares and high density hexagons at the highest density that we could access computationally. The phases coincide with the determined MECs (dashed line in Figure $5 \mathrm{e}$ ) and the experiments, with a quantitative agreement in the area fraction for the different phases between experiment (Figure 3, insets) and simulation (Figure $5 \mathrm{f}$, inset). The phases shown in Figure $5 \mathrm{f}$ could only be achieved through a very slow quench from high temperature (Methods), consistent with the observation that kinetic trapping can occur in the experimental system (Supplementary Figure 7). In this context, we also observe that the chain phase obtained from our simulations $\left(\rho r_{o}^{2}=0.75\right.$ in Figure $\left.5 \mathrm{f}\right)$ is less oriented than the chain phase obtained experimentally from dilating the system (Figure $3 \mathrm{f}$ ), but has a similar degree of orientational order compared to the chain phase obtained experimentally from compressing the system (Supplementary Figure $7 \mathrm{~d}$ ), indicating that the pre-orientation of the particles (as in the experiment under dilution) is responsible for chain alignment (compare Figure ze,f).

\section{Discussion}

The close agreement between theory, simulation and experiment supports the hypothesis that the observed assembly patterns result from a soft repulsive interaction potential, which we attribute to the presence of the microgels in the vicinity of the microspheres since pure microspheres did not show any complex assembly phases.

We rationalize the soft repulsive contribution to the interaction potential by the following effects. First, we observe that the PNiPAm microgels adsorb to the PS microspheres (Figure 1), similar to results reported in literature 44,49 . We further note that recent results from our group and others indicate attractive interactions between the PNiPAm microgels at the air/water interface ${ }^{50-52}$, which we also found for our microgels (Supplementary Figure 8). These attractive interactions prevent phase separation and lead to accumulation of microgels around the microspheres at the interface ${ }^{45}$. As a result, freely moving microspheres will be effectively repelled from each other, which we observe in the presence of microgels (Figure 2, Supplementary Figure 9). Importantly, the interparticle distance observed in the assembly phases suggests the presence of more than one layer of microgel around the particles, even after equilibration. We therefore rationalize that the combination of attractive microgelmicrosphere and attractive microgel-microgel interactions leads to the formation of a two-dimensional, disklike microgel corona around each microsphere at the interface. This picture is supported by both MECs and Monte Carlo simulations that accurately reproduce the observed phase diagram assuming such core-shell particles.

It is interesting to note that the complex phase behaviour at the air/water interface has not been observed for typical core-shell particles featuring a hard core surrounded by a soft, microgel shell. The only known phases for these types of colloidal particles are the hexagonal non-closepacked and close-packed phase 47,50,53. We attribute this clear difference in phase behaviour to the difference in the geometry of the "shell" component in our system (Figure 6). It is known that microgels deform at the interface into a three-dimensional oblate geometry 54,55 . As two deformed microgels are forced into closer proximity, their coronas start to overlap, inducing a repulsive interaction 55,56 . The repulsive potential for a given separation between two core-shell particles is to a first approximation proportional to the overlap volume between the coronas (Supplementary Discussion). The three-dimensional oblate shape of the microgels means that the overlap volume rapidly increases upon the approach of the two particles, which translates into a strongly concave interaction potential $(g<1)$ (Figure 6a). For such concave potentials, our model predicts only hexagonal structures as minimum energy configurations (Figure $5 \mathrm{c}$ ) with a transition between low density- and high density hexagonal phases, which is indeed found in experiment $47,50,53$. In contrast, in 


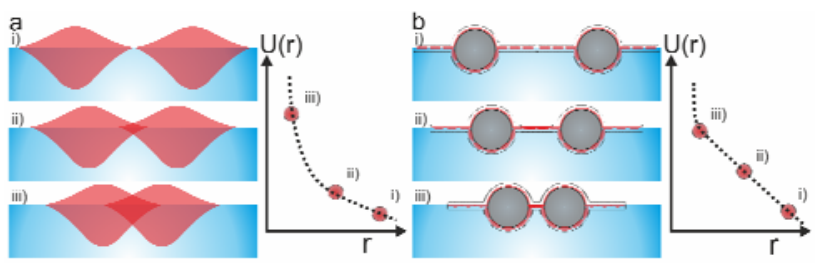

Figure 6. Graphical illustration of the hypothesized interaction potential between two microgel particles (a) and a mixture of small microgels and large microspheres (b) at the air/water interface. a) Interaction potential of two soft microgels. The microgels deform into an oblate shape at the interface (i). Upon approach, overlap of the microgels necessitates compression, which induces a repulsive interaction. The overlap volume of the coronas increases rapidly upon approach because of the three-dimensional nature of the microgels (ii-iii). Assuming the interaction potential is proportional to the overlap volume of the coronas, this yields an interaction potential that is strongly concave $(g<1)$ as illustrated on the right. b) Interaction potential of a mixture of microspheres and compressible microgels. After equilibration, the microspheres are surrounded by a two-dimensional disk-like corona of microgels at the interface (i). Upon approach, this $2 \mathrm{D}$ shell compresses, inducing a repulsive component similar to the case of a). However, with decreasing distance between the particles, the overlap volume of the coronas increases more slowly because of the twodimensional nature of the microgel corona (ii-iii). If to a first approximation we neglect the curvature of the disc going into the page, this results in an interaction potential that is approximately linear $(g \approx 1)$, as illustrated on the right.

our binary system, multiple individual microgels are accumulated around the microspheres at the air/water interface, forming a two-dimensional shell as discussed above. If to a first approximation we neglect the in-plane curvature of the corona, when two such core-shell particles approach each other under compression, the overlap volume between coronas increases approximately linearly $(g \approx 1)$ (Figure $6 \mathrm{~b})$. From the theoretical considerations (Figure 5), we find that $g \geq 1$ is a necessary condition to form the experimentally observed phases. The heuristic picture provided in Figure 6 may thus explain why the complex phase behaviour is only observed

for our two-dimensional core-shell particles but not for previously studied core-shell particles at liquid interfaces.If the repulsive component is caused by overlap of the microgel corona formed in situ at the air/water interface, the magnitude of the repulsive interaction, $U_{o}$ should relate to the elastic energy needed to compress the microgels. We first compute $U_{o}$ from the coexistence pressure $P_{\text {coex }}$ between the low density hexagonal phase and the chain phase as

$$
U_{o}=P_{\text {coex }}\left(A_{H E X L}-A_{C H}\right)
$$

where $A_{H E X L}$ and $A_{C H}$ are the area per particle in the low density hexagonal phase and chain phase respectively (Supplementary discussion, Supplementary Figure 10). We estimate the coexistence pressure from the experi- mental results as the pressure where there is a 50:50 mix of chains and low density hexagonal phase in the experiment as $P_{\text {coex }} \approx 26 \mathrm{mN} / \mathrm{m}$ (Figure 3 ) which yields $U_{o} \approx 4 \times 10^{-}$ ${ }^{14} \mathrm{~J}$ (Supplementary Discussion). We can independently estimate $U_{o}$ from the work done in elastically compressing the microgel coronas to fully overlap them. This yields

$$
U_{o} \sim P_{\text {microgel }} A_{\text {overlap }}
$$

where $P_{\text {microgel }}$ is the surface pressure (i.e., pressure per unit length) of microgels for typical surface concentrations found in the corona and $A_{\text {overlap }}$ is the overlap area of two disk-like coronas when their corresponding cores are touching (Supplementary discussion, Suppl. Figure 11). From the plateau in the surface pressure-area isotherm of pure microgels (Figure 2c), we estimate $P_{\text {microgel }}$ $\sim 28 \mathrm{mN} / \mathrm{m}$, yielding $U_{o} \sim 3 \mathrm{X10}^{-14} \mathrm{~J}$ (Supplementary Discussion) which is in excellent agreement with the calculation from the coexistence pressure. The agreement between the two results indicates that the soft repulsive shoulder in our system can arise from the elastic compression of an assembled, two-dimensional microgel layer around the microspheres.

\section{Conclusions}

In summary, we have demonstrated the possibility to manipulate the interaction potential of isotropic, spherical particles at an air/water interface by the addition of soft, compressible polymer microgels.

We observe a complex phase diagram as a function of the available area per particle at the interface, including wellaligned chains and square phases. Importantly, these unexpected phases arise from isotropic colloidal particles interacting via an isotropic interaction potential. The agreement of the experimental phase diagram with minimum energy calculations and Monte Carlo simulations at finite temperatures of spherical particles interacting leads to the conclusion that the microgels present at the air/water interphase induce a soft, repulsive component to the interaction potential of the microspheres. We attribute this additional, repulsive component to the formation of a two-dimensional microgel corona around the microspheres. From simple arguments of the shape of the particles at the interface, we further rationalize the shape of the soft repulsion shoulder and provide a tentative model why such complex assemble phases are not observed for typical core-shell particles. Finally, we find that the estimated magnitude of the repulsive shoulder is in excellent agreement with the energy required to fully overlap two microgel corona, indicating that elastic compression of a thin microgel layer at the interface can indeed give rise to the required soft shoulder repulsion. We anticipate this fundamental understanding to serve as a platform to engineer the shape and range of the interaction potential via the physicochemical properties of the microgels such as size and modulus, thus opening a rich phase space for the realisation of complex assembly structures. 
Our results provide general insights into the impact of interaction potentials on self-organization processes, demonstrating that surprising structural complexity can arise from the simplest possible building blocks and interactions. The approach greatly extends the available structural motifs available for surface patterning from simple building blocks, creating a powerful new platform for nanostructuration that is both fundamentally important and technologically useful.

\section{Experimental}

Experimental observation of the colloidal phases: Clean dispersions of polystyrene microspheres and poly $(\mathrm{N}-$ isopropylacrylamide) microgels (details in Suppl. Inform.) were diluted to $1 \mathrm{wt}-\%$ with a 1:1 ratio of ultrapure water and ethanol, mixed in an ultrasound bath, and spread onto a high compression Langmuir trough (KSV Nima) at room temperature until the surface pressure reached $\pi=26 \mathrm{mN} / \mathrm{m}$. The system was equilibrated by compression until all PS microspheres were in a hexagonal close packed phase $(\pi=34 \mathrm{mN} / \mathrm{m})$ and subsequently relaxed by barrier opening. After three closing-opening cycles, the phase diagram was recorded upon slow opening of the barriers $\left(3 \mathrm{~mm} / \mathrm{min}, 225 \mathrm{~mm}^{2} / \mathrm{min}\right)$. Image processing was done using Image J and analysed by a custom-written particle tracking software based on the publicly available IDL code by Crocker and Grier ${ }^{57}$. A detailed description of all experimental steps is provided in Suppl. Information.

Theoretical expression for enthalpy: The enthalpy per particle for the one particle unit cell shown in Figures(b) is given by Law et al. ${ }^{8}$

$$
H=\frac{1}{2} \sum_{n, m} U_{g}(|n \boldsymbol{a}+m \boldsymbol{b}|)+P a^{2} \gamma \sin \phi
$$

where the first term on the right hand side is the interaction energy between different core-shell particles while the second term is the pressure times the unit cell area. The lattice vectors are given by $\boldsymbol{a}=a(1,0), \boldsymbol{b}=\alpha \gamma(\cos \phi, \sin \phi)$ and $\alpha, \gamma$ and $\phi$ are the lattice constant, angle and aspect ratio of the unit cell respectively (compare Figure $5 \mathrm{~b}$ ).

The summation in the first term runs over all integer values of $n, m$ satisfying $|n a+m b| \leq \mathrm{r}_{\mathrm{c}}$ (except $n, m=0$ ), where $r_{c}$ is the cut-off radius for interactions, and the factor of $1 / 2$ corrects for double counting. Since the range of the shoulder repulsion is relatively small in our experimental system $r_{1} / r_{0}<2$, we choose $r_{c} / r_{o}=2$.

Monte Carlo Simulations: NVT Metropolis Monte Carlo simulations consisting of 1024 particles were performed in a rectangular box with aspect ratio $2: \sqrt{3}$ starting with the particles in a hexagonal lattice. For each density considered in Figure 5 (f), the particles were first disordered at $T^{*}=\infty$ (i.e., hard core repulsion only) and then brought to the final temperature through a very gradual quench of successive steps of $T^{*}=0.3,0.2,0.1,0.06,0.03,0.01$. At each step, the system was equilibrated for $10^{5}$ attempted moves per particle with an acceptance probability of around $30 \%$.

\section{ASSOCIATED CONTENT}

\section{Supporting Information.}

Supporting information is available free of charge on the ACS Publications website at http://pubs.acs.org."

Materials and methods, calculation of Uo, Supplementary figures including SEM, DLS and AFM characterisation, compression isotherm of the microgels, equilibration, different ratios and compression of the microsphere/microgel mixture, fluorescent microscopy images at the air/water interface and the geometry of the unit cell. Supplementary Movie 1 shows the phase transition between square and chain phase.

\section{AUTHOR INFORMATION}

\section{Corresponding Author}

*Email: nicolas.vogel@fau.de

*Email: d.m.buzza@hull.ac.uk

\section{ORCID}

Nicolas Vogel: orcid.org/oooo-0oo2-9831-6905

Marcel Rey: orcid.org/oooo-0oo2-1721-0253

\section{Funding Sources}

The research was supported by the Deutsche Forschungsgemeinschaft (DFG) under grant number VO 1824/6-1. N.V. also acknowledges funding of the Deutsche Forschungsgemeinschaft (DFG) through the Cluster of Excellence Engineering of Advanced Materials (EXC 315) and support by the Interdisciplinary Center for Functional Particle Systems (FPS).

\section{Notes}

The authors declare no competing financial interest.

\section{ACKNOWLEDGMENT}

We thank Roman Günther and Aukha Stoppa for their support on particle synthesis. We thank Prof. Siegfried Dietrich for discussions on step potentials and computational support.

\section{REFERENCES}

(1) McGorty, R.; Fung, J.; Kaz, D.; Manoharan, V. N. Mater. Today 2010, 13, 34-42.

(2) Binks, B. P. Curr. Opin. Colloid Interface Sci. 2002, 7, 2141.

(3) Wu, Y.; Wiese, S.; Balaceanu, A.; Richtering, W.; Pich, A. Langmuir 2014, 30, 7660-7669.

(4) Liu, T.; Seiffert, S.; Thiele, J.; Abate, A. R.; Weitz, D. a; Richtering, W. Proc. Natl. Acad. Sci. U. S. A. 2012, 109, 384-389.

(5) Binks, B. P.; Horozov, T. S. Angew. Chemie - Int. Ed. 2005, 44, 3722-3725.

(6) Gonzenbach, U. T.; Studart, A. R.; Tervoort, E.; Gauckler, L. J. Angew. Chemie - Int. Ed. 20o6, 45, 35263530.

(7) Kaz, D. M.; McGorty, R.; Mani, M.; Brenner, M. P.; 
Manoharan, V. N. Nat. Mater. 2011, 11, 138-142.

(8) Pieranski, P. Phys. Rev. Lett. 1980, 45, 569-572.

(9) Grzelczak, M.; Vermant, J.; Furst, E. M.; Liz-Marzán, L. M. ACS Nano 2010, 4, 3591-3605.

(10) Vogel, N.; Retsch, M.; Fustin, C. A.; Del Campo, A.; Jonas, U. Chem. Rev. 2015, 115, 6265-6311.

(11) Manoharan, V. N. Science 2015, 349, 1253751-1253751.

(12) Park, C.; Koh, K.; Jeong, U. Sci. Rep. 2015, 5, 8340.

(13) Kolle, M.; Salgard-Cunha, P. M.; Scherer, M. R. J.; Huang, F.; Vukusic, P.; Mahajan, S.; Baumberg, J. J.; Steiner, U. Nat Nano 2010, 5, 511-515.

(14) Boechler, N.; Eliason, J. K.; Kumar, A.; Maznev, A. A.; Nelson, K. A.; Fang, N. Phys. Rev. Lett. 2013, 111, 1-5.

(15) Rey, M.; Elnathan, R.; Ditcovski, R.; Geisel, K.; Zanini, M.; Fernandez-Rodriguez, M. A.; Naik, V. V.; Frutiger, A.; Richtering, W.; Ellenbogen, T.; Voelcker, N. H.; Isa, L. Nano Lett. 2016, 16, 157-163.

(16) Nemiroski, A.; Gonidec, M.; Fox, J. M.; Jean-Remy, P.; Turnage, E.; Whitesides, G. M. ACS Nano 2014, 8, 1106111070.

(17) Aveyard, R.; Clint, J. H.; Nees, D.; Paunov, V. N. Langmuir 2000, 16, 1969-1979.

(18) Isa, L.; Kumar, K.; Müller, M.; Grolig, J.; Textor, M.; Reimhult, E. ACS Nano 2o10, 4, 5665-5670.

(19) Lee, Y. H.; Shi, W.; Lee, H. K.; Jiang, R.; Phang, I. Y.; Cui, Y.; Isa, L.; Yang, Y.; Wang, J.; Li, S.; Ling, X. Y. Nat. Commun. 2015, 6, 6990.

(20) Geuchies, J. J.; van Overbeek, C.; Evers, W. H.; Goris, B.; de Backer, A.; Gantapara, A. P.; Rabouw, F. T.; Hilhorst, J.; Peters, J. L.; Konovalov, O.; Petukhov, A. V; Dijkstra, M.; Siebbeles, L. D. A.; van Aert, S.; Bals, S.; Vanmaekelbergh, D. Nat. Mater. 2016, 15, 1248.

(21) Gangwal, S.; Pawar, A.; Kretzschmar, I.; Velev, O. D. Soft Matter 2010, 6, 1413.

(22) Leunissen, M. E.; Vutukuri, H. R.; Van Blaaderen, A. Adv. Mater. 2009, 21, 3116-3120.

(23) Gong, T.; Wu, D. T.; Marr, D. W. M. Langmuir 2oo2, 18, 10064-10067.

(24) Yang, Y.; Gao, L.; Lopez, G. P.; Yellen, B. B. ACS Nano 2013, 7, 2705-2716.

(25) Froltsov, V. A.; Blaak, R.; Chremos, A.; Likos, C. N.; Loewen, H. J. Phys. Chem. B 2009, 113, 12316-12325.

(26) Osterman, N.; Babič, D.; Poberaj, I.; Dobnikar, J.; Ziherl, P. Phys. Rev. Lett. 2007, 99, 1-4.

(27) Cavallaro, M.; Botto, L.; Lewandowski, E. P.; Wang, M.; Stebe, K. J. Proc. Natl. Acad. Sci. 2011, 108, 20923-20928.

(28) Davies, G. B.; Krüger, T.; Coveney, P. V.; Harting, J.; Bresme, F. Adv. Mater. 2o14, 26, 6715-6719.

(29) Shindel, M. M.; Wang, S.-W.; Mohraz, A. Soft Matter 2012, 8, 6684 .

(30) Ershov, D.; Sprakel, J.; Appel, J.; Cohen Stuart, M. a; van der Gucht, J. Proc. Natl. Acad. Sci. U. S. A. 2013, 110, 9220-9224.

(31) Bianchi, E.; Capone, B.; Coluzza, I.; Rovigatti, L.; van Oostrum, P. D. J. Phys. Chem. Chem. Phys. 2017, 19, 19847-19868.

(32) Jagla, E. A. Phys. Rev. E 1998, 58, 11.

(33) Jagla, E. A. J. Chem. Phys. 1999, 110, 451-456.

(34) Glaser, M. A.; Grason, G. M.; Kamien, R. D.; Košmrlj, A.; Santangelo, C. D.; Ziherl, P. Europhys. Lett. 2007, 78, 46004.

(35) Dotera, T.; Oshiro, T.; Ziherl, P. Nature 2014, 506, 208211.

(36) Pattabhiraman, H.; Dijkstra, M. Soft Matter 2017, 13.
(37) Archer, A. J.; Rucklidge, A. M.; Knobloch, E. Phys. Rev. Lett. 2013, 111, 1-5.

(38) Torquato, S. Soft Matter 2oo9, 5, 1157.

(39) Zhang, G.; Stillinger, F. H.; Torquato, S. Phys. Rev. E Stat. Nonlinear, Soft Matter Phys. 2013, 88, 1-15.

(40) Malescio, G.; Pellicane, G. Nat. Mater. 2oo3, 2, 97-10o.

(41) Peng, Y.; Wang, F.; Wang, Z.; Alsayed, A. M.; Zhang, Z.; Yodh, A. G.; Han, Y. Nat. Mater. 2o14, 14, 1-16.

(42) Geisel, K.; Richtering, W.; Isa, L. Soft Matter 2014, 10, 7968.

(43) Zhao, C.; Yuan, G.; Han, C. C. Macromolecules 2012, 45, 9468-9474.

(44) Zanini, M.; Hsu, C.-P.; Magrini, T.; Marini, E.; Isa, L. Colloids Surfaces A Physicochem. Eng. Asp. 2017, 532, 116124.

(45) Louis, A. A.; Allahyarov, E.; Löwen, H.; Roth, R. Phys. Rev. E - Stat. Nonlinear, Soft Matter Phys. 2002, 65, 1-11.

(46) Vogel, N.; De Viguerie, L.; Jonas, U.; Weiss, C. K.; Landfester, K. Adv. Funct. Mater. 2011, 21, 3064-3073.

(47) Rey, M.; Fernández-Rodríguez, M. Á.; Steinacher, M.; Scheidegger, L.; Geisel, K.; Richtering, W.; Squires, T. M.; Isa, L. Soft Matter 2016, 12, 3545-3557.

(48) Yunker, P. J.; Chen, K.; Gratale, M. D.; Lohr, M. a; Still, T.; Yodh, a G. Rep. Prog. Phys. 2014, 77, 56601.

(49) Zhao, C.; Yuan, G.; Jia, D.; Han, C. C. Soft Matter 2o12, 8, 7036.

(50) Vogel, N.; Fernández-López, C.; Pérez-Juste, J.; Liz-Marzán, L. M.; Landfester, K.; Weiss, C. K. Langmuir 2012, 28, 89858993.

(51) Tsuji, S.; Kawaguchi, H. 2005, No. 15, 2434-2437.

(52) Rey, M.; Hou, X.; Tang, J. S. J.; Vogel, N. Soft Matter 2017.

(53) Rauh, A.; Rey, M.; Barbera, L.; Zanini, M.; Karg, M.; Isa, L. Soft Matter 2016, 13, 158-169.

(54) Geisel, K.; Isa, L.; Richtering, W. Langmuir 2012, 28, 1577015776.

(55) Geisel, K.; Isa, L.; Richtering, W. Angew. Chemie - Int. Ed. 2014, 53, 4905-4909.

(56) Style, R. W.; Isa, L.; Dufresne, E. R. Soft Matter 2015, 11, 1-8.

(57) Crocker, J. C.; Grier, D. G. Phys. Rev. Lett. 1996, 77, 18971900.

(58) Law, A. D.; Horozov, T. S.; Buzza, D. M. A. Soft Matter 2o11, $7,8923$. 


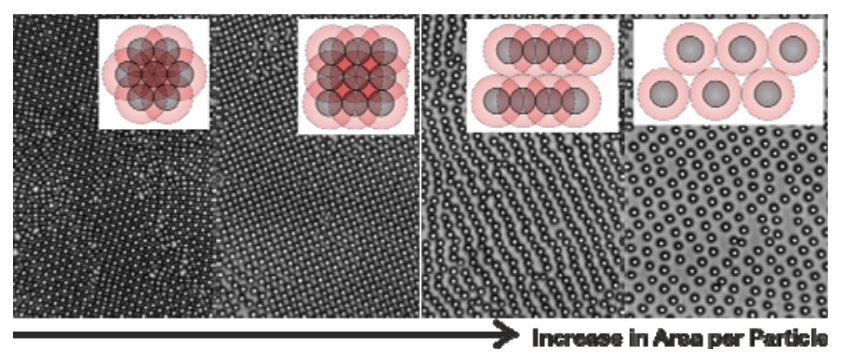

